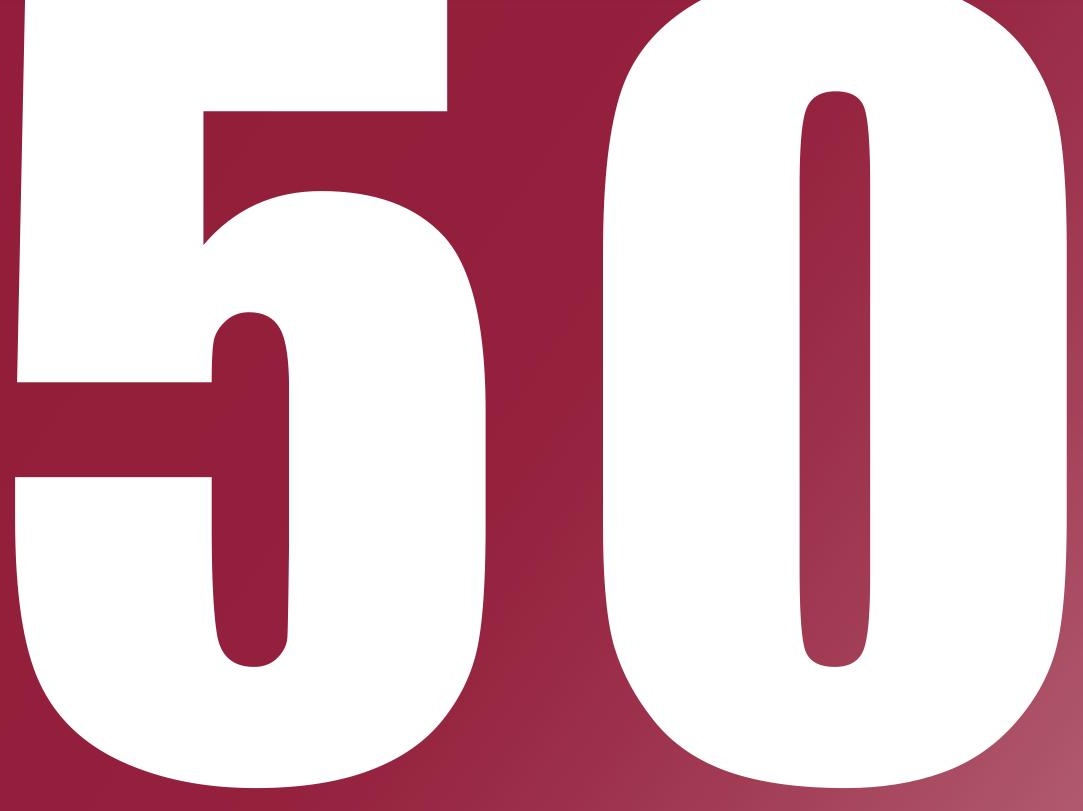

ÁMBITOS

REVISTA

INTERNACIONAL

DE

COMUNICACIÓN

$\mathbf{N}^{\circ} \mathbf{5 0}$

EDICIÓN OTOÑO

2020

ISSN: 1139-1979

E-ISSN: 1988-5733

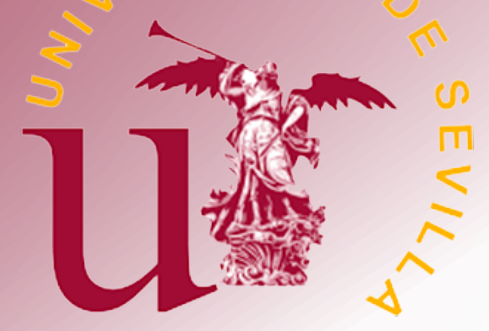




\section{INDICE}

EDITORIAL EDITORIAL

Presentación del Monográfico. Comunicación y juventud. Nuevos medios, representación, recepción y participación en contenidos de entretenimiento e información

Presentation of the monograph. Communication and youth. New media, representation, reception and participation in entertainment and information content.

Juan Francisco Gutiérrez Lozano, Francisco Javier Ruiz del Olmo

7-9

\section{MONOGRÁFICO MONOGRAPH}

El consumo audiovisual de la Generación Z. El predominio del vídeo online sobre la televisión tradicional The audiovisual consumption of Generation $Z$. The predominance of online video over traditional television

La imagen de los jóvenes en las estrategias transmedia de las series de televisión: el caso de Skam España en Instagram

The image of young people in transmedia strategies of TV series: the case of Skam España on Instagram

Sergio Jesús Villén Higueras, Francisco Javier Ruiz del Olmo

La penetración de Netflix en el público español ¿cuestiona el modelo televisivo tradicional?

Does the arrival of Netflix in the Spanish audience challenge the traditional television model?

Javier Bustos Díaz

49-61

Generación Z y consumo de información política: entre la televisión y los nuevos formatos mediáticos Generation $Z$ and consumption of political information: between television and new media formats

Ruth Gómez de Travesedo Rojas, Marta Gil Ramírez

$62-79$

Actualidad y comedia: El éxito de El Intermedio y La Resistencia entre los espectadores jóvenes

Information and comedy: The Success of El Intermedio and La Resistencia for young audiences

Inmaculada Concepción Aguilera García

80-95 
El efecto socializador de articular un espacio de comunidad virtual en el ecosistema del aula

The socializing effect of creating a virtual community space in the classroom environment

Lorea Ariadna Ruiz Gómez, Mónica Hinojosa Becerra, Francisco Javier Ruiz San Miguel

Juventude ciberativista e educação: reflexões sobre um jeito hacker de ser

Cyber activity youth and Education: reflections on a hacker way of being

Carla Azevedo de Aragão, Pietro Matheus Bompet Fontoura Alves, Karina Moreira Menezes

La risa grotesca en la obra de Bob Dylan: análisis de la Bacanal y el Festín Carnavalesco en la trilogía folk-rock eléctrica

Grotesque Laughter in Bob Dylan's Work: Analysis of The Bacchanal and the Carnival Feast in the Electric Folk-rock Trilogy

Jesús Albarrán Ligero

El papel del intertexto en el videojuego. Una partida, mil y una historias

Intertext in video games. A game, thousand and one stories

Rocío Serna-Rodrigo

145-158

\section{ÁmBitos PERSONALES PERSONAL ÁmBITOS}

El auge de Twitch: nuevas ofertas audiovisuales y cambios del consumo televisivo entre la audiencia juvenil The rise of Twitch: New audiovisual offers and the transformation of Television consumption among young audiences

\section{ARTÍCULOS ARTICLES}

La recepción de los medios de comunicación para residentes rusos en la Costa del Sol

Mass media reception by Russian residents on the Costa del Sol

Natalia Meléndez Malavé, José Carlos Pozo García

176-192

La calidad periodística en la cobertura de terremotos: Caso Ecuador

Journalistic quality in earthquake coverage: The case of Ecuador

Juan Pablo Arrobo-Agila, Mendoza María, Ignacio Aguaded

La credibilidad publicitaria en la nueva esfera mediática: los universitarios y los medios

Advertising credibility in the new media sphere: university students and the media 
Arco iris en medios brasileños: percepciones sociales sobre una campaña publicitaria LGBTQIA+

Rainbow in Brazilian media: social perceptions of an LGBTQIA+ advertising campaign

Leonardo Ferreira Batista, José Geraldo de Araújo Ramalho Filho

224-239

Rituales y comunicación política: la toma de posesión de los presidentes autonómicos españoles

Rituals and political communication: the Spanish autonomous Presidents inauguration

Ricardo Domínguez García

240-258

RESEÑAS REVIEWS

Una mirada diferente para hacer periodismo

A different look for making journalism

Guillermo Paredes-Otero

259-261

Periodismo y tecnología: una simbiosis continua

Journalism and technology: a continuous symbiosis

Luisa Graciela Aramburú Moncada

262-265

Una propuesta metodológica para impartir la asignatura de redacción periodística

Journalism and technology: a continuous symbiosis

Noelia Ruiz-Alba

266-268 


\title{
El efecto socializador de articular un espacio de comunidad virtual en el ecosistema del aula
}

\author{
The socializing effect of creating a virtual community space in the \\ classroom environment
}

\author{
Lorea Ariadna Ruiz Gómez \\ Universidad Complutense de Madrid | Calle Compañía 36, 2BB, Málaga 29008 | España \\ http://orcid.org/0000-0001-8030-3551 | lorearui@ucm.es
}

\section{Mónica Hinojosa Becerra}

Universidad Nacional de Loja | Calle Pichincha y Santa Mariana de Jesús, Urbanización El Prado, Casa no 2, Loja | Ecuador | http://orcid.org/0000-0003-3288-2135

| monica.hinojosa@unl.edu.ec

\section{Francisco Javier Ruiz San Miguel}

Universidad de Málaga | Calle León Tolstoi, 4, 2a planta, despacho 2. 65, Málaga 29071

| España | http://orcid.org/0000-0002-2210-0737 | sanmiguel@uma.es

Fechas | Recepción: 28/05/2020 | Aceptación: 16/09/2020 | Publicación: 15/10/2020

\section{Resumen}

El artículo trata de transmitir los primeros resultados obtenidos a través del Proyecto de Innovación Educativa de la Universidad de Málaga PIE19-018 "Simbiosis de aprendizaje formal e informal. El uso de la red social Instagram, como herramienta innovadora de debate y aprendizaje móvil colaborativo", emprendido en el curso académico 20192020. Instagram es la red social preferida por la generación Z y la que mayores incrementos de usuarios acumula en los últimos años. Se pretende desgranar algunos de los usos comunicativos desplegados en la experiencia, así como resaltar algunos de lo logros alcanzados. Todo ello de cara a plantear la

\section{Abstract}

The article tries to transmit the first results obtained through the Educational Innovation Project of the University of Malaga PIE19-018 "Symbiosis of formal and informal learning. The use of the social network Instagram, as an innovative tool for discussion and collaborative mobile learning", undertaken in the academic year 2019-2020. Instagram is the social network preferred by generation $Z$ and the one with the highest increases in users in recent years. It is intended to reel off some of the communicative uses deployed in the experience, as well as highlight some of the achievements achieved. All this in order to raise the discussion on the possibilities of the

\section{Forma de citar:}

Ruiz Gómez, L.A., Hinojosa Becerra, M. y Ruiz San Miguel, F.J. (2020). El efecto socializador de articular un espacio de comunidad virtual en el ecosistema del aula. Ámbitos. Revista Internacional de Comunicación 50, pp. 96-115. 
discusión sobre las posibilidades de la apropiación pedagógica de instrumentos ligados al consumo y a la producción de contenidos que no se enmarquen en el ámbito del entretenimiento. A estas intenciones se ha sumado la alarma sanitaria y el confinamiento por el COVID-19. El universo de estudio es 58 asignaturas repartidas en dos universidades españolas y cinco ecuatorianas. La muestra de estudio es la asignatura de Teoría y Análisis de la Imagen, del curso de 2019-2020, en la que participaron un total de 120 alumnos. El cuestionario se realizó desde el 1 hasta el 31 de marzo de 2020. A través de Instagram el proyecto está introduciendo nuevos sistemas de trabajo y estudio para el alumnado, implicando el conocimiento de TICs y la aplicación de dinámicas de difusión a través de Instagram. Se fomenta el discurso, la creación, la reflexión, el análisis, la retroalimentación y la defensa de las ideas.

Palabras clave: socialización, cultura digital, redes sociales, jóvenes, Instagram. pedagogical appropriation of instruments linked to consumption and the production of content that are not framed in the field of entertainment. To these intentions, the health alarm and confinement due to COVID-19 have been added. The universe of study is 58 subjects spread over two Spanish and five Ecuadorian universities. The study sample is the subject of Image Theory and Analysis, from the 2019-2020 course in which a total of 120 students participated. The questionnaire was carried out from March 1 to March 31, 2020. Through Instagram the project is introducing new work and study systems for students, involving knowledge of ICTS and the application of dissemination dynamics through Instagram. Discourse, creation, reflection, analysis, feedback and defense of ideas are encouraged.

Keywords: socialization, digital culture, social networks, young boys, Instagram.

\section{INTRODUCCIÓN}

En la sociedad contemporánea, dentro de los cambiantes límites de lo que se denomina juventud, se han ido parcelando las diversas etapas en función de las necesidades y planes de entrenamiento social tendentes a la producción. Se somete a las personas a la necesidad de una mutación en su estado, o se integran en el mundo del trabajo o dan el salto a la educación superior. En ambas opciones se genera un clima de incertidumbre que precisa de un tiempo de adaptación. Es en estas coyunturas vitales de inflexión en la socialización de los sujetos, en los ritos de paso, cuando el individuo busca un marco estable de autoafirmación y las redes sociales han posibilitado la existencia del espacio virtual, tan real y operativo para las relaciones personales como el mundo físico.

Durante el curso académico 2019-2020 hemos realizado una experiencia para discentes que ha consistido en emplear Instagram como un instrumento en el aula. Los objetivos del proyecto son exploratorios, aunque existen investigaciones previas (Moreno Moreno, 2018; Blanco Martínez y López Fernández, 2017). Se trata de testar las capacidades de Instagram como soporte para complementar la acción de enseñanza-aprendizaje desarrollada en el aula (Pérez-Rueda, Belanche y Lozano, 2019). También interesa detectar la capacidad de permeabilidad, a ampliar su tiempo e intensidad de dedicación a la interacción con la asignatura en espacios-tiempo externos a lo institucionalmente dispuesto para ello. Queremos sondear la capacidad de extender el interés por la asignatura fuera de los límites de lo establecido para su tratamiento 
formal. Como objetivo central queremos entender si las capacidades de socialización del alumnado pueden mejorar con el uso de Instagram.

Se trata de poder reflexionar en torno a la hipótesis de la capacidad facilitadora que la creación de un espacio virtual de comunicación e interrelación entre pares tiene para la socialización en el grupo de clase, incluso con el resto de grupos del mismo curso. La idea primera de emprender el proyecto nace de la búsqueda de respuestas a diversas incógnitas que preocupan al profesorado implicado en este estudio (Gómez Arruzazabala, 2019).

En un entorno social, económico e ideológico de alta competitividad; con un horizonte de futuro en el que los metarrelatos del contrato social modernista de la sociedad del bienestar han desaparecido o están en franca decadencia (Bauman, 2008); con directrices como las del Plan Bolonia, que alumbra la organización de los estudios en Europa, indicando que es preciso vincular el proceso de enseñanza/aprendizaje a la futura empleabilidad (Riesco González, 2008, p. 81), pero en un mundo que tiende a la pérdida masiva de puestos de trabajo y a la mutación vertiginosa de las labores reclamadas en los entornos laborales que persistan en un futuro cercano (Adsuara Varela, 2015; Ramos \& Rodríguez, 2015; Schwab, 2017; Vidal, 2015) ¿Qué estrategias emplear para alcanzar un grado aceptable de motivación en el cuerpo discente?, ¿cómo potenciar el nivel de implicación y participación del alumnado en la materia de la asignatura?, ¿cómo generar un ambiente más propicio para el trabajo colaborativo?, ¿qué herramienta implementar para generar una mayor conciencia de comunidad? Las cuestiones que se plantean son complejas, pero quizás vertebradoras de la labor cotidiana en el aula, en la relación ternaria docente-materia-discente.

El planteamiento general de la experiencia toma en consideración su desarrollo en cuatro fases consecutivas, desde el punto de vista de los docentes implicados en la misma, ya que se cubre la repetición del ensayo durante los dos semestres de dos cursos académicos consecutivos (2019-2020 y 2020-2021). Debemos señalar que los acontecimientos de la pandemia de COVID19 va a trastocar todo este plan, al tener temporalmente paralizada toda la actividad docente en los dos países implicados en el estudio: España y Ecuador.

Desde el punto de vista de los discentes, el experimento tan solo tiene extensión durante el curso académico y dado que el uso de la cuenta de Instagram se circunscribe a su uso en una asignatura y estas tienen un desarrollo semestral, para ellos el desarrollo se lleva a cabo durante el periodo de docencia y evaluación de las asignaturas implicadas (Ruiz-San-Miguel et al., 2020).

Desde esta perspectiva y tratando cada asignatura como una unidad de ensayo, se diseñó una programación de acciones, observaciones y recogida de datos secuencial, continua, que permitiese diluir al máximo la tentación de interpretaciones subjetivas, que potenciarían la falta de rigor en el conocimiento obtenido, así como las posibles dispersiones de sentido respecto a los objetivos concretos planteados. Pero que también permitiese un nivel de flexibilidad suficiente para poder tomar en consideración posibles ajustes durante su realización.

Al trabajo previo de revisión bibliográfica en torno a las diversas facetas implicadas en el estudio (juventud, características generacionales, redes sociales y su uso), se le fueron añadiendo una 
serie de herramientas metodológicas, cuantitativas y cualitativas, que durante el desarrollo de la labor de campo permitieran recoger el mayor número de datos, y que estos resulten significativos de cara a poder construir unas conclusiones que no solo den respuesta a las incógnitas planteadas en origen, sino que permitan trazar nuevas cuestiones que abran y complementen la línea de investigación emprendida.

Para la recogida de datos de la muestra compuesta por el alumnado de dos grupos grandes y siete grupos reducidos, de dos asignaturas, una del Grado de Comunicación Audiovisual y otra de Historia del Arte, especialidad en Museología, se han empleado dos encuestas, secuenciadas en el tiempo, la observación participante a lo largo de la experiencia y la recogida y procesado de toda la información que procura la propia aplicación, en cuanto a la actividad de los discentes en ella.

La primera acción planificada, a desarrollar en la última sesión de trabajo presencial de la semana de presentación de la asignatura, consiste en la realización de la primera de las encuestas, compuesta de 41 preguntas centradas en el conocimiento, consumo, generación de contenidos y costumbres de los discentes respecto al entorno y ecosistema digital, sus herramientas y los usos que tienen rutinizados.

Esta encuesta se realiza de manera anónima a través del campus virtual, en una de las aulas informáticas provistas en las instalaciones de la facultad, de manera sincrónica para todos los participantes y con una limitación de tiempo para cumplimentarla. Estas especificaciones no son arbitrarias, sino dictadas por el afinamiento de la técnica obtenido durante los últimos once cursos académicos, en los que ya se ha estado realizando con otros fines y objetivos, en grados y niveles variados. Y tiene la función de que la muestra sea lo más amplia posible, y alcance el mayor grado de fidelidad con la realidad del conocimiento de cada encuestado, al buscar que su realización sea individual y fiable.

Realizar el pase de este cuestionario tiene como fin último tratar de concretar el conocimiento y alfabetización digital del alumnado implicado en cada caso, con el afán de complementar la visión de los perfiles generacionales que se aportan en la literatura generada en torno al concepto de juventud como colectivo, que gira alrededor de las generaciones de los "nativos digitales", centrada la atención en aquellos designados como "Generación Z" (nacidos entre 1994 y 2010), lapso de edad en el que se enmarcan todos aquellos discentes con los que vamos a trabajar durante estos dos cursos académicos.

Con los datos obtenidos y tras su revisión, se puede validar el resto de pasos diseñados o reajustarlos a las posibilidades y necesidades del conjunto de los encuestados. Una vez cotejados los datos de la encuesta inicial, se procede a la apertura de la cuenta en el servicio de la red social Instagram.

El perfil, con el nombre de la asignatura implicada, es cerrado, solo accesible para los miembros activos de la misma, lo que permite generar un espacio híbrido, público para todos los miembros, pero privado fuera del ámbito de la asignatura. Ello genera un nivel de control adecuado para permitir la flexibilidad suficiente que proporcione una percepción de libertad 
expresiva, preservando los derechos e intimidad dentro de los límites de la comunidad de la clase, y minimice riesgos, sin sacrificar la creatividad y la capacidad comunicativa. Este ambiente controlado busca construir un marco de confortabilidad y confianza relacional, que ayude a la creación de lo que proponemos denominar cuarto espacio (Oldenburg y Brissett, 1982), ubicado en la esfera virtual, pero de forma híbrida con el resto de espacios donde se desarrolla el conjunto del proceso de enseñanza-aprendizaje.

Desde el perfil de la asignatura en Instagram, se despliega, después de su presentación al alumnado y de su incorporación voluntaria a la misma, una estrategia de comunicación combinada, que atienda a los propósitos marcados de inicio, ordenados por bloques de capacitaciones.

Atenderemos, con las piezas multimedia aportadas, a las necesidades-objetivos de contenidos de la materia, contribuciones que coadyuven a la socialización, propuestas que incentiven la motivación y participación, y diversos aportes que puedan catalizar la creación de un cierto espíritu de comunidad alrededor de la asignatura.

En el primer bloque, el dedicado a los temas, tanto teóricos como prácticos de la asignatura, se procede a publicar imágenes fijas (dibujos, esquemas o fotografías), vídeos y textos, todos ellos con el fin de:

- Reforzar los contenidos vistos en el aula, por la posibilidad de su repetición, aunque con otra narrativa diferente, que pueda aportar un cierto grado de interés para su visita.

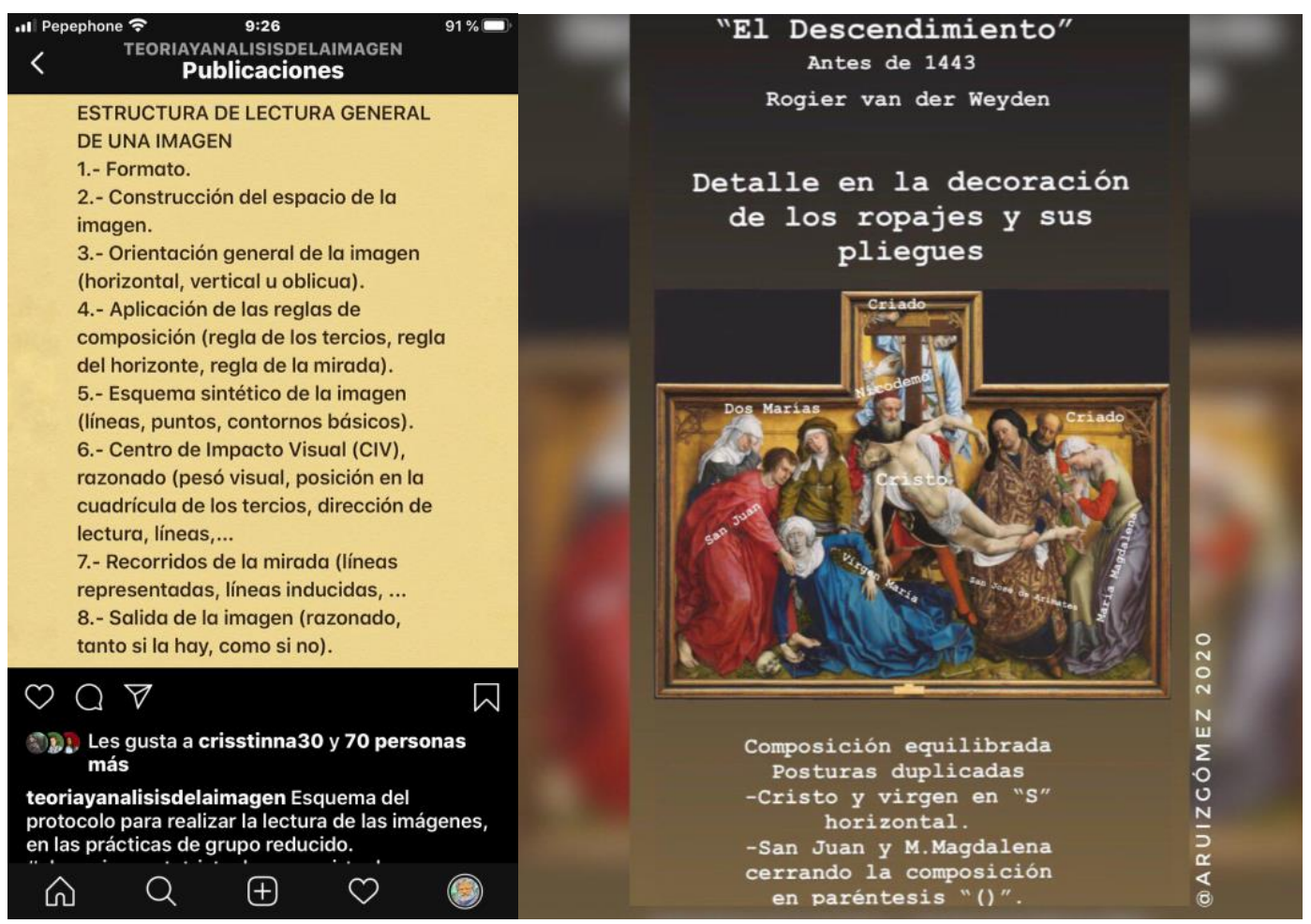

Figuras 1 y 2. Contenidos de refuerzo en dos asignaturas

Fuente: elaboración propia de los autores. 
- Ampliar los contenidos con ejemplos y performances-experiencias vividas en el aula. Hay que tomar en consideración que en la organización de las enseñanzas en base a grupos grandes y reducidos, las actividades desarrolladas en el seno de estos últimos son reiterativos en los tres o cinco grupos reducidos por asignatura.

- Ampliar la calidad de la experiencia sensorial de la vivencia del proceso de enseñanzaaprendizaje más allá del espacio personal vivido en el aquí-ahora del aula. La posición de cada persona en el aula imprime una experiencia parcializada, a veces incompleta y otras distorsionada, tanto a nivel visual y auditivo, sin hacer referencia a la posible influencia de otras percepciones sensoriales no tomadas en consideración, por no reparar cognoscitivamente en ellas al asumir la naturalidad de su inmanencia a la cotidianeidad del aula, pero que tienen una influencia, como puede ser el exceso de frio o calor que hace incomoda la estancia y distrae la atención, el grado de ruido de fondo, los olores y densificación de la atmósfera del espacio con el transcurrir del tiempo en la clase, todo elementos que quedan minimizados, eliminados, con la puesta en escena de los contenidos a través del cuarto espacio, lo que permite al discente tener una experiencia nueva y complementaria, que además le permite replicarla, revivirla cuantas veces necesite.

- Asentar y ordenar los puntos fuertes de cada apartado de la materia, estableciendo esquemas sintéticos que, no solo sirvan como mojones que marcan el final de la impartición de un apartado, sino como verdadera "chuleta" siempre a mano, que permita su consulta, en la realización de los ejercicios prácticos realizados en el aula. En lo que respecta al orden de los conceptos, viene muy apoyado por el propio orden establecido por la aplicación en los contenidos aportados, que siempre es de más novedoso a más antiguo, al que los discentes están acostumbrados por su uso de herramientas que emplean esa sistematización de las aportaciones.

En cuanto al bloque de contribuciones que coadyuven a la socialización:

- Nominación de los miembros de la comunidad, para crear el marco de relación entre personas, como individuos singulares y únicos. En este sentido no es determinante que la nominación responda al nombre real de los sujetos que interactúan, sino que comenzará por sus "apodos" en la red social que acepta de buen grado y con el que se encuentra cómoda. Con la desvirtualización en el segundo espacio (en este caso el entorno aula-universidad), de forma natural, se pasará a la nominación más personal y/o se mantendrá la adoptada en la Red. Hay que tomar en consideración este aspecto, ya que desde la niñez se asocia la nominación institucional a través del uso del apellido, en detrimento de la basada en el nombre de pila, lo que es percibido como un cierto grado de despersonalización.

- Construir cooperativamente y publicar contenidos de relación entre los diversos grupos en los que están estructuradas las enseñanzas, para crear un primer contacto de conocimiento y hacer a todos copartícipes de la existencia del cuarto espacio común. 
- Aportar contenidos de mera relación, que sirvan de tarjeta de presentación entre subgrupos, o bien que muestren el trabajo colaborativo, de ayuda o complemento entre las acciones, deberes y responsabilidades de esas diversas agrupaciones.

Propuestas que incentiven la motivación y participación:

- Potenciar la atención y la puesta en valor de las singularidades de los miembros individuales de la comunidad, alentando la aportación de materiales, informaciones, ideas, proyectos a través de la comunicación que facilita la cuenta común de la red social.

- Dosificar mensajes de exigencia, de cara a la realización de las tareas, pero siempre apoyados por una contraparte de transmisión de ánimo y refuerzo positivo.

- Transversalidad en la difusión de un tono empático en el conjunto de las comunicaciones, con especial atención al uso de argumentos, expresiones y lenguajes inclusivos y que respeten y promuevan la diversidad y el valor que para el desarrollo de la relación en la comunidad de la asignatura tienen la expresión, creatividad y aportaciones personales de los individuos que forman el colectivo.

- Introducir un grado de gamificación en el proceso de enseñanza aprendizaje, con el fin de fijar la atención sobre conceptos y estructuras del conocimiento visitado, así como fomentar la participación e implicación, con la consecuencia de asentar todo ello a través de la construcción del conocimiento en base a la acción concreta. Este objetivo se ha vehiculado a través de breves encuestas de autoevaluación propuestas a través de Instagram. 


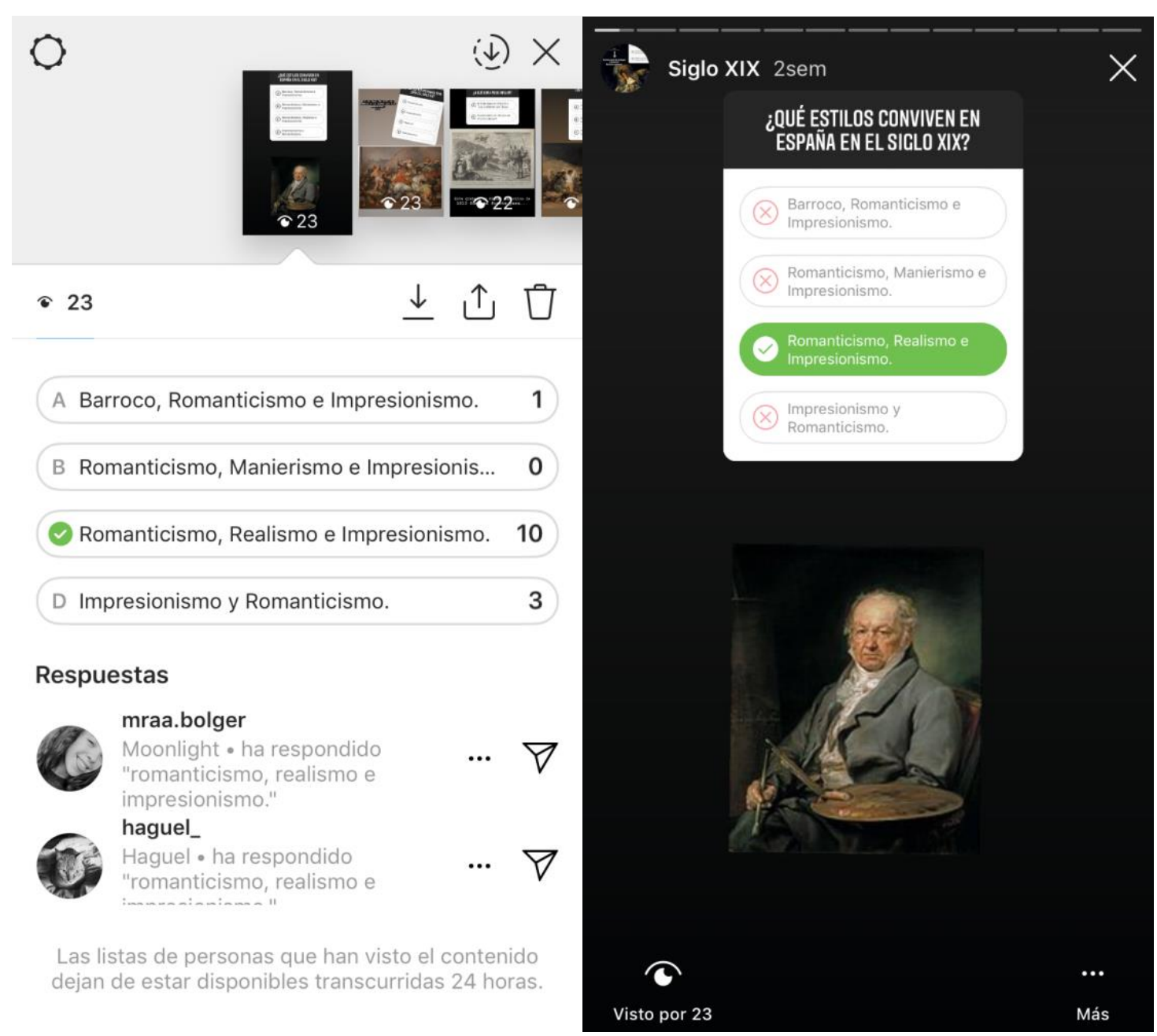

Figuras 3 y 4. Contenidos de gamificación para repaso de materia Fuente: elaboración propia de los autores.

Aportes tendentes a la creación de percepción de comunidad y de cuarto espacio en el seno de la asignatura:

- Buscar la transversalidad en los contenidos generados, para que tengan presencia en ellos la mayor variedad de grupos implicados en la comunidad y de los sujetos individuales que los conforman.

- Introducir, en todos aquellos aportes que no estén incluidos dentro de los apartados de contenidos de conocimiento, un cierto nivel de comunicaciones de carácter menos formal, que puede vehicularse a través de elementos lingüísticos o paralingüísticos, siempre con respeto, que permita pequeños respiros de distensión, que favorezcan la percepción de una experiencia confortable y de confianza en el cuarto espacio generado en el ámbito virtual. Es interesante testear con antelación el tipo de contenidos, lenguaje, elementos paralingüísticos, opciones de la herramienta y usos que mejor se adapten al conjunto de los miembros de la asignatura y que mayor consenso puedan 
despertar. Para ello puede resultar muy provechosa la observación de las cuentas personales de los discentes y las formas de relación entre ellos.

- Contenidos circunscritos a compartir la libre expresión en torno a acontecimientos programados, cíclicos o espontáneos, con el fin de ritualizar la puesta en común de valores positivos, y de surtir a la comunidad de pequeños hitos de vivencias menos institucionales, pero con gran valor de comunión y conocimiento entre los miembros del grupo.

Se solicita la opinión de los discentes a través de una encuesta final, compuesta de 28 preguntas, estructurada en varios bloques temáticos que permitan conocer y medir el grado de satisfacción del alumnado respecto a la experiencia, así como permitir que aporten su opinión para mejorarla en las siguientes ediciones. La organización de estos bloques temáticos puede resumirse de la siguiente manera: preguntas en torno al uso de la red social empleada; a las motivaciones primordiales para participar en la experiencia; percepción subjetiva del beneficio e implicación que ha supuesto para la materia, para los individuos y para el grupo; el nivel de interés y motivación a la participación que ha generado cada una de los diversas aportaciones; la percepción de intromisión en la intimidad que ha podido suponer el uso de la cuenta en el entorno de la asignatura; el interés o deseo por el abandono o permanencia como miembro de la cuenta una vez finalice el curso académico; y un espacio para las sugerencias de mejora y las críticas que ayuden a afinar y mejorar el uso de la herramienta.

\section{REVISIÓN TEÓRICA Y PLANTEAMIENTO DEL PROBLEMA}

Podríamos emprender una larga recapitulación de definiciones y modelos de comunicación, pero quizás ninguna de las primeras más sencilla, concreta, sincrética que la formulada por Gerbner (1967). Y complementando su punto de vista y siempre bajo la guía de las preguntas fundadoras que nos hemos planteado, acudir a la observación de las dos concepciones alternativas de comunicación que vienen planteándose desde el comienzo del interés por su estudio y que parten de dos perspectivas y supuestos epistémicos diferentes, una visión de la comunicación como transmisión y una visión de la comunicación como ritual (Carey, 2009, p. 12), como relación.

Ambas contienen con acierto partes de la naturaleza del fenómeno, pero obedecen a dos planteamientos que se apoyan en bases muy diferentes y que buscan resultados contrapuestos y que, en el ámbito del proceso de enseñanza-aprendizaje tiene una importancia capital, pues dan soporte a dos formas muy encontradas de entender estos procesos.

Así, la comunicación concebida como transferencia, como transmisión de señales mediante un código común al emisor y al receptor (Real Academia Española, 2018), es deudora de las tradiciones saussuriana y estructuralista (Baylon y Mignot, 1996, p. 231), con lo que ello conlleva de la búsqueda del logro de un uso estandarizado de la comunicación que consiga que todos los individuos reproduzcan los mismos signos para los mismos conceptos (Saussure, Bally, Sechehaye, y Riedlinger, 1985, p. 29). Esto supone una puesta en suspenso de todo lo que pueda 
suponer una autonomía para los sujetos que actúan en la comunicación, lo que en el ámbito de la comunicación pedagógica supone la literal extirpación de las singularidades personales de los individuos, para tratar de ajustarlos a un estándar medio. Esto nos remite a su vez, a los modelos comunicativos coetáneos de la imposición de esta visión de la comunicación como mera transmisión, en los cuales nos encontramos con su base conductista, basada en el binomio causal de estímulo-respuesta, cuya transcripción en la enseñanza decimonónica sería el clásico acción-reacción.

Esta concepción de los procesos de comunicación como procesos de transmisión, también tiene como consecuencia la desapropiación de cualquier incidencia en él del contexto social en el que se desarrolla, alejando así toda asociación con el valor etimológico del vocablo "comunicar", del latín communicāre, que a su vez deriva de communis, común, mutuo, participado entre varios (Real Academia Española, 2018).

Y es en este punto donde nos encontramos con la otra concepción del fenómeno, la visión ritual de comunicar, la perspectiva relacional, que desde las preguntas que nos formulamos inicialmente, $y$ desde los objetivos a los que ellas nos abocan, es el marco cognitivo que más se ajusta a nuestras necesidades y apetencias. En ella, nos movemos en el ámbito del compartir con otros, de la participación, de la asociación, de la posesión de ideas, intereses y propósitos comunes. Adoptando esta visión de la comunicación en general y de la relación comunicativa entre docente y discentes en particular, trascendemos la mera transmisión de los mensajes en el espacio y el tiempo para acceder, como señala Carey, a la representación de aspiraciones y creencias compartidas que se emplean para la construcción de un ámbito cultural ordenado que puede servir como control para la acción (Carey, 2009, p. 15).

John Dewey lo aclara centrando nuestra atención en conceptos relacionados con algunas de nuestras aspiraciones expresadas, cuando asevera que las personas conviven en una comunidad en virtud de las cosas que tienen en común; y la comunicación es la forma en que llegan a poseer cosas en común. $Y$ esas cosas en común han de ser objetivos, creencias, aspiraciones, conocimiento, una comprensión común, una mentalidad similar (...). El consenso exige comunicación (Dewey, 1916, pp. 5-6). Esta perspectiva ritual, relacional, concibe la comunicación como lugar de encuentro y expresión de las subjetividades (Baylon y Mignot, 1996, pp. 231-232).

La experiencia se lleva a cabo en asignaturas de diversos grados y en diversos niveles de estos. Nos encontramos con personas entre los 17 y 22 años. Para los dos cursos académicos en los que se implementa el proyecto, interaccionaremos con discentes nacidos entre 1998 y 2004. Nos encontramos ante miembros de la denominada "Generación Z".

Trataremos de dibujar un breve bosquejo en torno a aquellas posibles características más justificadas por los contextos sociales, económicos, políticos y sobre todo tecnológicos y de consumo-creación de contenidos que pueden envolver a nuestros interlocutores en esta experiencia. 
Como señalan algunas autoras Popescu, Popa y Cotet (2019, p. 243), se ha investigado más sobre cómo se debe abordar la Generación Y y la Generación $Z$ en el lugar de trabajo que en cómo deben abordarse en el aula. Los estudios realizados están mostrando que los docentes tienen menos conocimientos sobre las características de esta generación (Correia Barreiro y Bozutti, 2017, p. 156), que recibe también otras denominaciones como postmillenial, centennial, posbieber, iGeneration, Gen Z, Generación K, N-GEN o Generación en Red, D-GEN o Generación Digital (Correia Barreiro y Bozutti, 2017; Dias, Caro, y Gauna, 2015; Díaz Caneja, 2019; Montaña Blasco, 2018).

Nos encontramos ante la primera generación que puede denominarse "nativa digital" (Espiritusanto, 2016, p. 112), si tomamos en consideración la fecha de aparición del primer navegador, Mosaic, en 1993, para su uso sobre la recién nacida World Wide Web (WWW) de Tim Berners-Lee, de 1991. Y si establecemos como siguiente fecha de inflexión la de la puesta en funcionamiento del motor de búsquedas más usado, Google, en 1998.

Los miembros de esta generación tienen una notable inclinación hacia el uso de tecnologías, dispositivos, herramientas, aplicaciones y servicios digitales. No en vano han crecido en un ambiente cotidiano de acceso a Internet, a través de Wi-Fi y con una considerable exposición al uso del teléfono móvil inteligente como herramienta universal. Un estudio de Universia (2015) señalaba el alto grado de dependencia de la población universitaria en cuanto al uso de este dispositivo. Del total de entrevistados, más de la mitad, el 51\%, se declaraban dependientes del móvil. Actualmente el mismo porcentaje alcanza ya al conjunto de la población, en todos los segmentos de edad.

La tecnología y la accesibilidad es de suma importancia para los componentes de esta generación, de manera que no conciben la posibilidad de un mundo sin conexión, ya que a través de las TIC satisfacen necesidades de entretenimiento, diversión, comunicación, información e, incluso, formación (Dias et al., 2015, p. 1).

En cuanto a sus preferencias por las herramientas para comunicarse, en dispositivo hardware es el Smartphone, y por lo que respecta a las aplicaciones, las redes sociales (Dias et al., 2015, p. 3), con preferencia por las audiovisuales, principalmente YouTube e Instagram.

La cotidianización de su dependencia tecnológica les permite una notable capacidad multitarea y multipantalla, pudiendo procesar información de varias fuentes, en hasta cinco dispositivos de manera simultánea, como señalan las profesoras Inma Rodríguez-Ardura y Neus Soler (Sánchez Mateos, 2018).

Se muestran más conscientes de su intimidad que otras generaciones contiguas, más contenidos en sus intercambios en las redes sociales (Llaneza, 2016), aunque presenten rasgos más abierto a nivel social (muestran una clara inclinación al activismo social y al voluntariado) (Page, 2018), e individual (una identidad sexual más dúctil y libre de tabús) (Duffy et al., 2018).

En el ámbito de su preparación, tanto su inmersión digital como las características de inestabilidad e inseguridad económica y el ambiente de alta competencia imprimen en ellos un 
carácter autodidacta que les empuja a intentar emprender desde muy jóvenes (Llaneza, 2016), o buscar empleos que no les aten en exceso, les permitan creatividad e interrelación, aunque todo ello acompañado con un grado de impaciencia y permanencia de atención (Díaz Caneja, 2019).

Con una coyuntura por la crisis a la que se sometió al sistema educativo, especialmente la enseñanza superior, al confluir la implantación del nuevo Espacio Europeo de Enseñanza Superior tras el desplome financiero de 2008, y con las secuelas de los recortes presupuestarios, que infradotaron la implantación de los nuevos planes de nivel de grado, los miembros de la generación recién llegada a la universidad muestran cierto recelo hacia el sistema de enseñanza tradicional, lo que les impulsa a dotarse de otras herramientas para acceder al aprendizaje, lo que deriva en un escoramiento hacia materias más escogidas desde un punto de vista vocacional-emocional, con una fuerte carga de interés hacia las experiencias (Espiritusanto, 2016, p. 112).

Todo esto abona el terreno para la introducción de una herramienta como Instagram, de cara a conformar un nuevo marco de relación en el proceso pedagógico. Y a ello ayudan además los datos de uso de Internet y redes sociales en España. Según consta en el informe anual de redes sociales iab de 2019 (ELOGIA, 2019, p. 6 y 8), de los 30,9 millones de españoles entre 16 y 65 años, el $92 \%$ es internauta y el $85 \%$ usuarios de redes sociales.

Pero para poder percibir la idoneidad de la herramienta elegida, señalar que Instagram creció en España un 49\%, durante el 2019, con una implantación del 70\% entre personas de 16 a 30 años (ELOGIA, 2019, p. 18). Y que las funcionalidades que nos proporciona nos permiten trabajar con material textual, icónico, audiovisual, incluso un canal de televisión donde incluir videos de larga duración, además facilita la interactuación directa, pero también el seguimiento del grupo y de cada uno de los participantes en él.

El universo de estudio es 58 asignaturas de nueve grados o carreras distintas, y cinco asignaturas de dos posgrados diferentes (máster universitario). Todo ello en dos universidades españolas (Universidad de Málaga y Universidad Complutense de Madrid) y cinco ecuatorianas (Universidad Nacional de Chimborazo, Universidad de Guayaquil, Universidad Nacional de Loja, Universidad Técnica Particular de Loja y Pontificia Universidad Católica del Ecuador, sede Ibarra). Esto nos va a permitir evaluar y comparar los resultados en los ámbitos culturales de ambas realidades iberoamericanas. La muestra de estudio es la asignatura de Teoría y Análisis de la Imagen, del curso de 2019-2020. Participaron un total de 120 alumnos. El cuestionario se realizó desde el 1 hasta el 31 de marzo de 2020.

\section{RESULTADOS}

Los frutos del ensayo que se presentan en esta aportación son parciales, tanto por el escaso tiempo transcurrido desde su comienzo, como por el impedimento que está suponiendo la alarma sanitaria mundial del Covid-19. Consideramos que los resultados ya son sustanciales y con un claro valor prospectivo, de cara a orientar el resto de la experiencia. 
La investigación se realiza de manera secuencial, continua y acumulativa, con lo que los datos que se van obteniendo en cada una de las acciones, se ordenan y analizan, para poder sacar conclusiones parciales que ayuden a validar o flexibilizar las fases ulteriores.

En los datos recogidos con la encuesta inicial no se dieron sorpresas sobre los resultados previstos, tanto por la experiencia acumulada durante cursos anteriores, como por lo manifestado por algunos expertos (Burin, Coccimiglio, González, y Bulla, 2016; Cabra-Torres y Marciales-Vivas, 2009; Dans, 2017; Flores Alarcia y del Arco Bravo, 2013; Ramirez de León y Ruiz, 2019), que advierten de cómo la brecha generacional ha creado ciertos lugares comunes que no resultan sino ideas estereotipadas, como es el mito de los "nativos digitales" (Prensky, 2001) que la realidad de los datos contradice.

Los factores de los que más nos interesaba conseguir información a través de este primer cuestionario eran aquellos que nos ayudasen a construir un perfil colectivo en lo tocante a:

- El nivel de alfabetización digital. Y los indicadores nos reafirman en los resultados que venimos obteniendo durante toda esta década, que demuestran la falsedad del mito de los "nativos digitales" y su alto grado de conocimiento digital. Se trata de un saber operativo, desestructurado en lo tocante a la adquisición de información y producción de conocimiento. Con un conocimiento limitado en el tipo de herramientas digitales que se pueden emplear en la Red (el 93,39\% no conoce ni usa aplicación de agregación de contenidos; el 98,35\% no conoce o usa marcadores sociales, tampoco conocen gestores bibliográficos y tan solo un $63,64 \%$ conoce o usa la herramienta de intercambio y trabajo colaborativo asociada a la cuenta de Google).

- Los rasgos denotan que sí predominan actitudes de consumo digital, destacando cierta propensión a la actividad "prosumer". Al destacar un perfil consumidor, a través de un número limitado de herramientas, la mayoría de ellas orientadas por ellos para el consumo de productos audiovisuales (casi el $80 \%$ no tiene blog donde volcar sus aportaciones y de los que lo tienen, el 93,39\% declaran no publicar con regularidad).

- El nivel de uso/dependencia de Internet (tiempo de uso, dispositivos prioritarios, espacios de uso). El $100 \%$ posee Smartphone, con servicio de datos por encima de $1 \mathrm{~Gb}$ al mes, con acceso a Internet por encima de las tres horas diarias $(54,55 \%$ entre tres y cuatro horas, y casi el $43 \%$ con más de cuatro horas diarias).

- En cuanto al interés y uso en las redes sociales, señalar que casi el $90 \%$ les dedican por encima de las dos horas diarias (45,45\% más de tres horas al día). Y entre ellas destaca Instagram. 


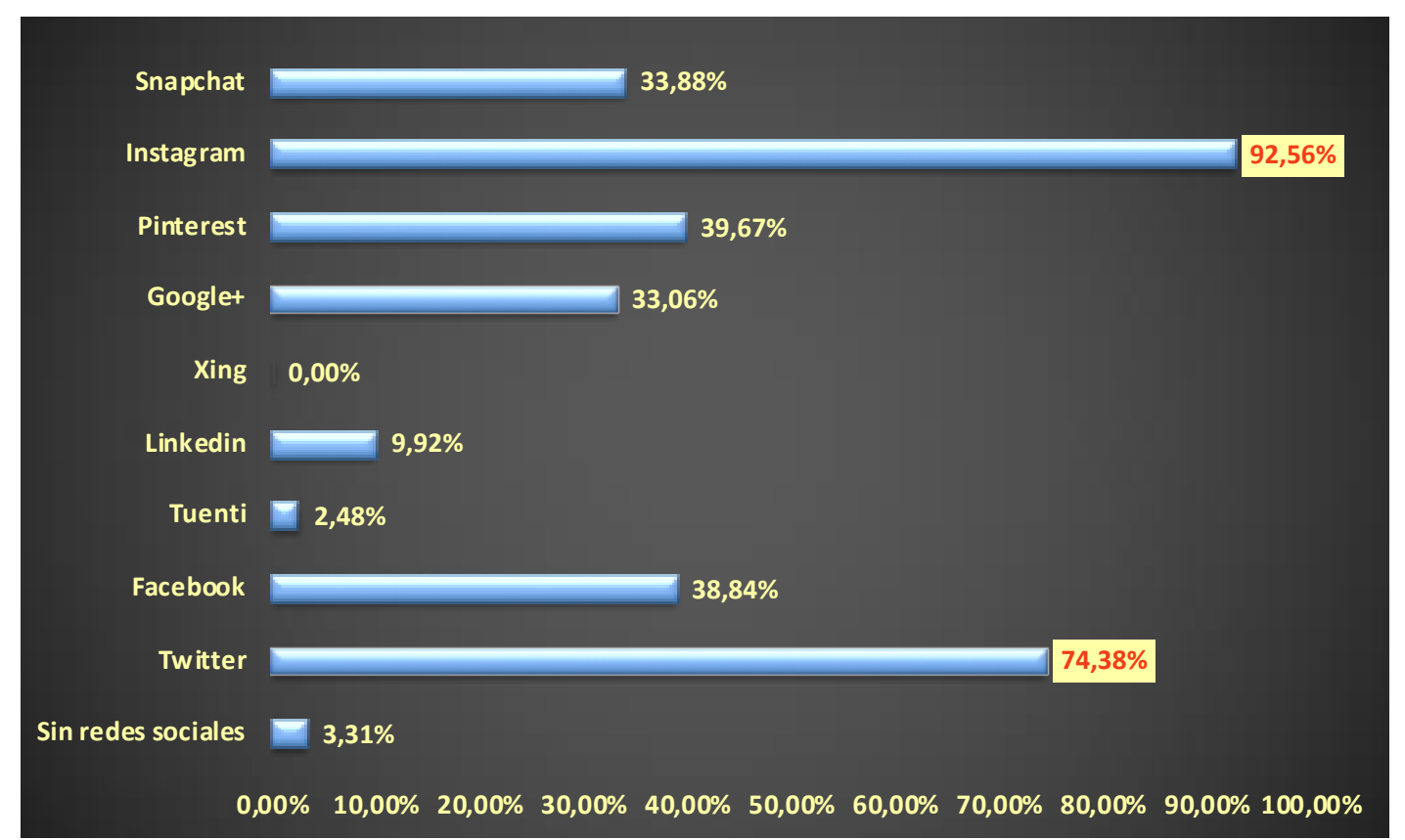

Figura 5. Participación de los discentes en redes sociales

Fuente: datos recogidos en la encuesta inicial de la experiencia.

En cuanto a los resultados arrojados por la propia experiencia, tanto en referencia a los recogidos y analizados durante su transcurso, como a los obtenidos con el cuestionario realizado al alumnado tras la finalización del periodo de la asignatura, lo primero es que los receptores de la experiencia la están acogiendo positivamente. Por la novedad que para ellos supone, el 94,9\% no había empleado nunca Instagram para fines académicos y, de todas las posibles razones que les movieron a participar en el ensayo, las dos primeras apuntaban a implementar su conocimiento de la materia y la curiosidad. 


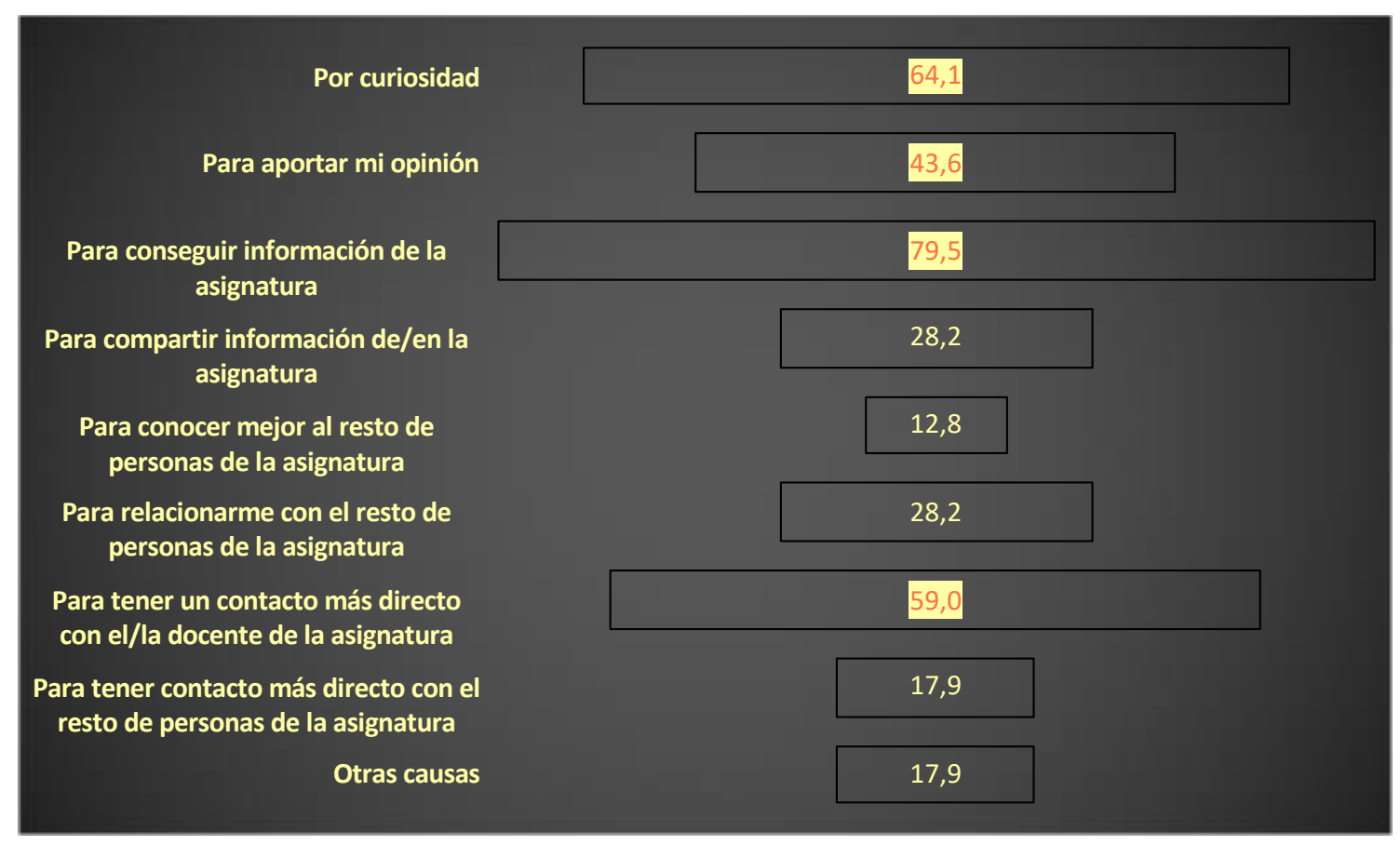

Figura 6. Argumentos de participación voluntaria en la experiencia Fuente: datos recogidos en la encuesta final de la experiencia.

Por lo que respecta a la consecución de objetivos en cuanto al apoyo de la transmisión de contenidos de la asignatura, si los instrumentos metodológicos empleados pueden quedar validados por los resultados obtenidos, debemos tener una percepción positiva de las herramientas y contenidos generados, ya que el $85,5 \%$ de los discentes han superado su asignatura, con casi el $50 \%$ de ellos con calificaciones de notable o sobresaliente. Creemos que la incidencia que los contenidos generados a través de Instagram han podido tener influencia en la materia.

Tabla 1

Resultados obtenidos en la evaluación de las asignaturas (1. ${ }^{a}$ convocatoria)

\begin{tabular}{|l|c|}
\hline \multicolumn{1}{|c|}{ Variables observadas } & Porcentaje sobre total \\
\hline Discentes que superan la asignatura & $85,5 \%$ \\
\hline Discentes con calificación de notable o superior & $48,6 \%$ \\
\hline Discentes que no superan la materia & $9,4 \%$ \\
\hline Discentes que finalmente no se han presentado / no evaluados & $4,3 \%$ \\
\hline
\end{tabular}

Fuente: datos recogidos de las actas de las asignaturas implicadas en la experiencia. 
Todos los contenidos circunscritos a la materia de la asignatura, aportados en vídeo, tanto a través de "stories" de 24 horas y posterior almacenamiento en el apartado específico de destacados, como el canal IGTV, han sido visitados por el conjunto de los discentes, y revisitados, hasta alcanzar una media del $120 \%$ de visitas.

En el apartado de los resultados de promoción de la participación y motivación del alumnado, quizás es donde mejores resultados se han recogido. Señalar, por ejemplo, que en la relación discente-docente se ha incrementado la fluidez si la comparamos con la habitual que se da sobre los soportes institucionales habituales, como correo electrónico y campus virtual. Solo en los datos de la comunicación a través de mensajes directos, el $70 \%$ del conjunto ha interaccionado con el profesorado, con un mínimo de dos conversaciones por persona, a un máximo de 35 diálogos.

Tabla 2

Resultados más significativos, para los objetivos de motivación y participación

\begin{tabular}{|l|c|}
\hline \multicolumn{1}{|c|}{ Variables observadas } & Resultados \\
\hline Porcentaje medio de visitas realizadas al material de la cuenta Instagram & $120 \%$ \\
\hline $\begin{array}{l}\text { Porcentaje de discentes que han interactuado con el docente (Mensajes } \\
\text { directos) }\end{array}$ & $70 \%$ \\
\hline Intensidad de interacción discentes/docente (no de conversaciones/discente) & Entre 2 a 35 \\
\hline
\end{tabular}

Fuente: datos recogidos de los resultados de la actividad en la cuenta de Instagram.

Por lo que respecta a la percepción directa que el alumnado ha tenido de la experiencia, el $97,4 \%$ considera que el uso de esta herramienta ha sido beneficioso para el desarrollo de la asignatura, calificándola todos ellos con un grado de beneficio de 4,7 sobre una escala de 5. A nivel individual, el $94,9 \%$ declara que le ha ayudado a interesarse más por la asignatura, con un nivel de interés medio de 4,2 sobre 5 .

En cuanto a la percepción de los discentes, un $84,6 \%$ considera que el uso de Instagram le ha impulsado a implicarse en la asignatura, con un nivel medio de 3,9 sobre 5. La implicación del resto de compañeros la perciben como alta un $94,9 \%$, valorándola con un 4,2 sobre 5 , mientras que en el caso del tutor en la experiencia, el 97,4\% la perciben con un nivel de implicación de 4,9 sobre 5 .

Tabla 3

Resultados más significativos, para los objetivos de motivación y participación

\begin{tabular}{|l|c|}
\hline \multicolumn{1}{|c|}{ Variables observadas } & Resultados \\
\hline Inclusión de la experiencia percibida como beneficio para la asignatura & $97,4 \%$ \\
\hline Nivel de beneficio de la experiencia para la asignatura & 4,7 sobre 5 \\
\hline
\end{tabular}




\begin{tabular}{|l|c|}
\hline $\begin{array}{l}\text { Inclusión de la experiencia percibida como factor de incremento de interés para la } \\
\text { materia }\end{array}$ & $97,4 \%$ \\
\hline Nivel de incremento de interés para la materia & 4,7 sobre 5 \\
\hline Percepción de implicación personal en la materia & $84,6 \%$ \\
\hline Nivel de implicación personal en la materia & 3,9 sobre 5 \\
\hline Percepción de implicación grupal en la materia & $94,9 \%$ \\
\hline Nivel de implicación grupal en la materia & 4,2 sobre 5 \\
\hline Percepción de implicación del docente en la materia & $97,4 \%$ \\
\hline Nivel de implicación del docente en la materia & 4,9 sobre 5 \\
\hline
\end{tabular}

Fuente: datos recogidos de los resultados de la encuesta final.

\section{DISCUSIÓN Y CONCLUSIONES}

La investigación aún se encuentra en su fase inicial y durante lo que resta de estos cursos aún se podrá ir ampliando. Tanto desde el punto de vista de resultados, del material obtenido para su análisis y reflexión, como en la generación de pautas que puedan guiar el trazado de un marco de actuación, promete un cierto nivel de productividad, que esperamos implementar a nivel de experiencia y creación de conocimiento sobre la materia.

Los primeros resultados cualitativos y cuantitativos son positivos. Está favoreciendo la implicación colectiva del alumnado y también presenta las bases metodológicas para incorporar de forma permanente dicha actividad como desarrollo de la teoría de forma alternativa a las clases y envío de tareas prácticas en la asignatura de Teoría y Análisis de la Imagen. Una vez establecido y adaptado el modelo para su ejecución dentro de los parámetros de la asignatura, se plantean la posibilidad de incorporar, en los próximos años, este diseño de desarrollo de teoría y actividades prácticas de uso de Instagram proponiendo al alumnado nuevas herramientas que fomenten un acercamiento a la materia de estudio.

La primera conclusión es que debemos desterrar la idea de que los discentes de las generaciones denominadas "nativos digitales" están en posesión de una alfabetización digital competente y suficiente. Nos enfrentamos a un prejuicio generacional, apoyado en el desconocimiento y temor tecnológico promocionado por la brecha de edad. Nos encontramos ante un colectivo de alumnado con unas altas destrezas operativas en un limitado número de herramientas digitales, empleadas para el consumo de productos audiovisuales, con un bajo perfil "prosumer".

Hay que subrayar la curiosidad e implicación que muestran, con un alto nivel de exposición en la Red, a través de las redes sociales y con dedicación a la comunicación relacional, en un entorno de hibridación de espacios y con una vivencia temporal de inmediatez. Se trata de personas conectadas, con un alto grado de implicación en el diálogo virtual, sobre soportes audiovisuales. 
Todas estas apreciaciones implican una coyuntura muy favorable para la inclusión de Instagram como herramienta de apoyo en el aula. Entendemos que los puntos fuertes de la inclusión de esta herramienta se centran alrededor de aquellas tendencias que hemos destacado en el perfil general de las personas con las que se va a interactuar.

Para el discente resulta muy atractivo que la contraparte del diálogo educativo le hable en su canal preferente, en su plaza global, donde ha elegido estar, con un lenguaje similar y conocido, que agiliza y personaliza la relación y que permite una disponibilidad permanente del material, en el espacio y en el tiempo. Más que un aula sin muros, un espacio de relación de enseñanza/aprendizaje extendido en espacio y tiempo.

\section{Referencias}

Adsuara Varela, B. (2015). ¿En qué trabajarán mis hijos? El auto-empleo y el emprendimiento en la Economía Digital. Revista de estudios de juventud, (108), 103-123.

Bauman, Z. (2008). Los retos de la educación en la modernidad líquida. Barcelona: Gedisa.

Baylon, C., \& Mignot, X. (1996). La comunicación. Madrid: Cátedra.

Blanco Martínez, A. y López Fernández, R. (2017). La posibilidad pedagógica de la red social Instagram para aproximar a los estudiantes al género poético. En Ruiz-Palmero, J., Sánchez-Rodríguez, J. y Sánchez-Rivas, E. (Edit.). Innovación docente y uso de las TIC en educación. Málaga: UMA Editorial. https://bit.ly/37GW8ul

Burin, D., Coccimiglio, Y., González, F., \& Bulla, J. (2016). Desarrollos recientes sobre Habilidades Digitales y Comprensión Lectora en Entornos Digitales. Psicología, Conocimiento y Sociedad, 6(1), 191-206. https://bit.ly/3bRc80p

Cabra-Torres, F., \& Marciales-Vivas, G. P. (2009). Mitos, realidades y preguntas de investigación sobre los «nativos digitales»: Una revisión. Universitas Psychologica, 8(2), 323-338. https://bit.ly/3bRQc5A

Carey, J. W. (2009). A Cultural Approach to Communication. En Communication as culture (pp. 11-28). New York: Routledge.

Correia Barreiro, S., \& Bozutti, D. F. (2017). Challenges and Difficulties to Teaching Engineering to Generation Z: a case research. Propósitos y Representaciones, 5(2), 127-183. https://doi.org/10.20511/pyr2017.v5n2.163

Dans, E. (2017, febrero 26). Más sobre esos nativos digitales que no existen. ED Enrique Dans. https://bit.ly/2ZvMeul

Dewey, J. (1916). Democracy and education an introduction to the philosophy of education. New York: The Macmillan Company.

Dias, C. B., Caro, N. P., \& Gauna, E. J. (2015). Cambio en las estrategias de enseñanza-aprendizaje para la nueva Generación Z o de los "nativos digitales". Virtual Educa, 1-21. https://bit.ly/3hnvvzJ

Díaz Caneja, J. P. (2019). La Generación Z en el lugar de trabajo en España. https://bit.ly/3k9z5iM

Duffy, B., Thomas, F., Shrimpton, H., Whyte-Smith, H., Clemence, M., \& Abboud, T. (2018). Beyond Binay. The lives and choices of Generation Z. Ipsos MORI. https://bit.ly/3bQll2U

ELOGIA. (2019). Estudio anual de Redes Sociales IAB 2019. Madrid: iab spain. https://bit.ly/32kK5DU

Espiritusanto, N. (2016). Generación Z: móviles, redes y contenido generado por el usuario. Revista de Estudios de Juventud, (114), 111-126. 
Flores Alarcia, Ó., \& del Arco Bravo, I. (2013). Nativos digitales, inmigrantes digitales: Rompiendo mitos. Un estudio sobre el dominio de las TIC en profesorado y estudiantado de la Universidad de Lleida. Bordón. Revista de Pedagogía, 65(2), 59-74. https://bit.ly/2Ffaqg4

Gerbner, G. (1967). Mass Media and Human Communication Theory. En F. E. X. Dance (Ed.), Human Communication Theory (pp. 40-60). New York: Holt, Rinehart y Winston.

Gómez Arruzazabala, G (2019). Instagram para el fomento de la Educación Física. Lecturas: Educación Física y Deportes, 24(254), 89-100. 2019. https://bit.ly/32fWzuM

Llaneza, P. (2016). La Generación Z: incógnitos y privados. Revista de Estudios de la Juventud, (114), 143156.

Montaña Blasco, M. (2018). La nueva generación Z: conectada, pragmática y familiar. Comein. Revista de los Estudios de Ciencias de la Información y de la Comunicación, (77).

Moreno Moreno, M.P. (2018). Uso docente de la red social 'Instagram' en la asignatura de Proyectos 1. En VI Jornadas sobre Innovación Docente en Arquitectura (JIDA'18), Escuela de Ingeniería y Arquitectura de Zaragoza, 22 y 23 de Noviembre de 2018. Universitat Politècnica de Catalunya. Iniciativa Digital Politècnica. DOI10.5821/jida.2018.5511

Oldenburg, R., \& Brissett, D. (1982). The third place. Qualitative Sociology, (5), 265-284. https://doi.org/10.1007/BF00986754

Page, B. (2018, julio). Generation Z - Beyond Binary: new insights into the next generation. Ipsos MORI. https://bit.ly/32sVION

Pérez-Rueda, A.; Belanche, D. \& Lozano, N. (2019). Instagram como herramienta de aprendizaje en el aula universitaria. En IN-RED 2019: V Congreso de Innovación Educativa y Docencia en Red. https://bit.ly/2HM1SJa

Popescu, D., Popa, D. M., \& Cotet, B. G. (2019). Getting ready for Generation Z students - considerations on 3D printing curriculum. https://doi.org/10.20511/pyr2019.v7n2.280

Prensky, M. (2001). Digital Natives, Digital Immigrants. On the Horizon, 9(5), 1-7. https://bit.ly/3ikphSx

Ramirez de León, M. R., \& Ruiz, O. (2019). El mito de los nativos digitales, tendencias en la educación superior. Facultad de Arquitectura. Universidad de San Carlos de Guatemala. https://doi.org/10.13140/RG.2.2.16819.27688

Ramos, J., \& Rodríguez, C. (2015). Cambio Tecnológico y Hegemonía Económica: el papel de las TICs en la Configuración del Nuevo Orden Económico Mundial. Revista de estudios de juventud, (108), 89102. https://bit.ly/2FnVI4h

Real Academia Española. (2018). Diccionario de la lengua española. Recuperado de https://dle.rae.es

Redacción noticias.universia.es. (2015, octubre). El 51\% de los jóvenes españoles se considera dependiente del móvil. Noticias Universia España. Universia. https://bit.ly/3kb8xO1

Riesco González, M. (2008). El enfoque por competencias en el EEES y sus implicaciones en la enseñanza y el aprendizaje. Tendencias Pedagógicas, (13), 78-105. https://bit.ly/3irDkWj

Ruiz-San-Miguel, F. J., Ruiz Gómez, L. A., Hinojosa-Becerra, M., \& Maldonado-Espinosa, M. (2020). Uso de Instagram como herramienta de debate y aprendizaje. 2020 15th Iberian Conference on Information Systems and Technologies (CISTI). DOI: 10.23919/CISTI49556.2020.9140998

Sánchez Mateos, A. (2018, julio 15). 10 datos sobre la Generación Z. La Vanguardia. https://bit.ly/32q8YOF

Saussure, F. de, Bally, C., Sechehaye, A., \& Riedlinger, A. (1985). Curso de lingüística general. Barcelona: Planeta. 
Ruiz Gómez, L.A., Hinojosa Becerra, M. y Ruiz San Miguel, F.J.

Schwab, K. (2017). La cuarta revolución industrial. Barcelona: Debate.

Vidal, M. (2015). Un mundo automático. Revista de estudios de juventud, (108), 69-87.

Ámbitos. Revista Internacional de Comunicación | ISSN: 1139-1979 | E-ISSN: 1988-5733, №. 50. (2020) 